\title{
Air in- and exfiltration through the joints of external walls
}

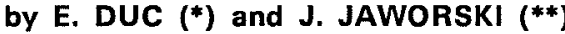

(*) Technical University of Wroclaw, Institute of Building Engineering, Poland.

$\left.{ }^{(* *}\right)$ Polish Academy of Sciences, Institute of Fundamental Technological Research, Poland.

\begin{abstract}
This paper presents a new method of investigating and checking airtightness of the joints of external walls by means of a thermovision system. This typical investigation is very efficient and more accurate as compared to that of the pressure method. The investigation of airtightness was performed on joints with the simple cracks which were extra made by cutting off and polishing typical sandwich walls. The two half-sandwich walls were joined up or framed into one, to conduct the airtightness test for the crack. The obtained frame was mounted (fixed) in the empty external window-frame. The room was situated in the half height of a few story building. The instruments for measuring of the climate (radiation, pressure, temperature, air speed, etc.), blower door with ventilators (regulating the pressure difference across the joint), and thermovision equipment (880 LWP + TIC 8000) were used. In the calculation, of temperature fields in unsteady state the programme BLOW-CRACK for temperature bridge with crack was used.
\end{abstract}

\section{Numerical model and stability solution}

The typical thermal effect of airtightness of buildings is presented in papers [1] and [2]. Assuming that the thickness crack $h_{s} \simeq h$, where $h$ is the nextwork step in space with two-dimensional mesh, and that the joint of external walls is on the axis crack (figure $1, \mathrm{~b}$ and $\mathrm{c}$ ), when the material is homogeneous (thermal conductivity $\lambda$, specific heat capacity $c$, density $\rho$ ), general balance equation for filtration (leakage of air) is defined as follows:

$$
\begin{aligned}
\vartheta_{i, j, n+1} & =\frac{\Delta \tau}{h^{2} c \rho}\left\{(\lambda+A) \vartheta_{i, j-1, n}+\left[\frac{h^{2} c \rho}{4 \Delta \tau}-(\lambda-B)\right] \vartheta_{i, j, n}+(\lambda-C) \vartheta_{i, j+1, n}\right. \\
& \left.+\left[\frac{h^{2} c \rho}{4 \Delta \tau}-(\lambda+D)\right] \vartheta_{i, j, n}+\frac{\lambda \alpha_{s} h}{\left(\alpha_{s} h+\lambda\right)} \vartheta_{i-1, j, n}+\left[\frac{h^{2} c \rho}{2 \Delta \tau}-\frac{2 \lambda \alpha_{s} h}{\left(\alpha_{s} h+\lambda\right)}\right] \vartheta_{i, j, n}+\frac{\lambda \alpha_{s} h}{\left(\alpha_{s} h+\lambda\right)} \vartheta_{i+1, j, n}\right\}
\end{aligned}
$$

with: $\vartheta_{i, j, n+1}$ temperature in the axis crack at time $(n+1) \Delta \tau,{ }^{\circ} \mathrm{C} ; \Delta \tau$ elementary time interval for computation (time step), $\mathrm{s} ; \alpha_{s}$ convective heat transfer coefficient in the crack, W. $\mathrm{m}^{-2} \cdot \mathrm{K}^{-1}$.

Equation (1) presents three variants infiltration description and three variants for exfiltration depending on the accepted values of $A, B, C, D$. For example, when infiltration moves accordingly in the accepted direction of $j$, we choose or take the first variant, where air temperature which enters the element is equal to the temperature of the node backwords $(i, j-1)$ and in the exit - the temperature of central node $(i, j) ; A=2 \alpha_{p}, B=-A, C=D=0$.

Coefficient of heat transfer in the crack $\alpha_{p}\left(W \cdot \mathrm{m}^{-1} \cdot \mathrm{K}^{-1}\right)$ is given by:

$$
\alpha_{p}=I_{z} c_{a} \rho_{a} / 2
$$

with: $I_{z}$ air permeability in joint, $\mathrm{m}^{2} \cdot \mathrm{s}^{-1} ; \quad c_{a}$ specific heat capacity of air, $\mathrm{J} . \mathrm{kg}^{-1} \cdot \mathrm{K}^{-1} ; \rho_{a}$ density of air, $\mathrm{kg} \cdot \mathrm{m}^{-3}$.

The second variant, where the air temperatures of the inlet and outlet of element are equal to the middle temperature, the central node and the neighbouring, adequate backward and next forwards takes out when $A=B=C=D=\alpha_{p}$. 


\section{http://dx.doi.org/10.21611/qirt.1992.049}

In the case of exfiltration, air flow direction is opposite to the direction of $j$. For example, the third variant, where the air temperature entering the elements is equal to the temperature in the node, that is next $(i, j+1)$ and in the exit - the temperature in the central node $(i, j)$, demands putting into equation (1): $A=B=0, C=-2 \alpha_{p}, D=-C$.

Assuming that the change of temperature along crack axis (near the central node) is linear, the use of one of three variants (for infiltration or exfiltration) is limited to non-zero word in the round brackets, e.g., at temperature $\vartheta$, see equation (1). The initial result of the analysis allows to use for infiltration the first variant and third variant for exfiltration.

The investigation, of the stability, e.g., of the first variant of infiltration continues as follows. The value of $\Delta \tau$ and $h$ may be determined or qualified as independently of every direction out of four heat flow directions or for four altogether. Assuming the adequate small value of $h$, the stability condition in the following time step [equation (1)] requires the error calculation not to increase. From the algebraic sum in the square brackets which cannot be equal to zero, we conclude the following limitation:

$$
\Delta \tau_{\max }<h^{2} c \rho\left[2 \lambda+2 \lambda \alpha_{s} h /\left(\alpha_{s} h+\lambda\right)+2 \alpha_{p}\right]
$$

This is the criterion used in this paper.

In the regions outside the crack, the classical equation balance [3], is used except of the nodes situated at the distance $h$ from the surface crack, where the temperature is described by the following equation:

$$
\begin{aligned}
\vartheta_{i-1, j, n+1} & =\vartheta_{i-1, j, n}+\Delta \tau\left[\lambda \left(\vartheta_{i-1, j-1, n}+\vartheta_{i-1, j+1, n}+\vartheta_{i-2, j, n}-3 \vartheta_{i-1, j, n}\right.\right. \\
& \left.+\lambda \alpha_{s} h\left(\vartheta_{i, j, n}-\vartheta_{i-1, j, n}\right) /\left(\alpha_{s} h+\lambda\right)\right] /\left(c \rho h^{2}\right)
\end{aligned}
$$

For a two-dimensional homogeneous mesh, the stability condition [3] is:

$$
\Delta \tau \leqslant h^{2} \mathrm{c} \rho(4 \lambda)
$$

\section{Experimentation}

The experiment was conducted on the basis of equations (1) - (5). The simulation of a joints airtightness, whose thickness of cracks were 0.001 and $0.002 \mathrm{~m}$ considering different pressure (across the joints) and different temperatures for both the warm and cold sides of the walls, proved that the changes of temperature effects can be registered by thermovision system. The testing of airtightness of the joint without a seal, for different thickness of cracks, was carried out on the windward side of the building. The weather was cloudy in the day. The investigation of the joint was conducted when external air temperature was approximately zero degrees centigrade.

The door of the room was replaced with a blower door the hot air from the entrance hall and staircase to let blow into the room. The temperature on the cold and warm sides of the joint, air temperature difference for both sides, speed air in the joint and the pressure difference across the joint were recorded.

The experiment was carried out in three states. The first state - the natural conditions, the second state - the transient ones and the third state - the forced ones.

In the natural state there was only the thermal effect caused by wind pressure on the windward (cold) side (infiltration through the crack). In the transient state, gradual (step-by-step) motion of fans system in the blower door was used. This caused the pressure in the room to be higher than the pressure on the windward side of the wall and air flow through crack outside (exfiltration). In the forced state, the effect of temperature on the cold and the warm sides stabilized. 


\section{Experimental results}

Ideal airtightness joint on the interior side must have a temperature $\vartheta_{i, s}^{*}$ equal to the temperature on the warm side of the wall $\vartheta_{i}^{*}$ far from the joint. Resulting from the air infiltration with on air speed $v=0.3 \mathrm{~m} / \mathrm{s}$ through the crack thickness $h_{s}=0.001 \mathrm{~m}$ (for internal temperature $t_{b}=20$ and external air temperatute $t_{e}=-0.4^{\circ} \mathrm{C}$ ), the temperature on the warm side of the joint $\vartheta_{i, s}^{*}$ lowers to $9.7^{\circ} \mathrm{C}$, while the temperature far from the joint $\vartheta_{i}^{*}$ was averaged to $19.6^{\circ} \mathrm{C}$ (curve 0 in figure $1 \mathrm{~d}$ ). For the the external measurements, the difference temperatures on the joint and surface, which may be the base for analysis of airtightness, was not detected by the thermovision system.

At the moment, when infiltration changes to exfiltration, after one hour, the air speed in the crack $v=0.24 \mathrm{~m} / \mathrm{s}$ and air temperatures: $t_{i}=21$ and $t_{e}=0^{\circ} \mathrm{C}$. The temperature on the warm surface joint $\vartheta_{i, s}^{*}$ grows and reaches locally the value of the surface temperature $\vartheta_{i}^{*}$ far from the joint. After $0.5 \mathrm{~h}$ continuation, $\vartheta_{i, s}^{*}=20.5$ and $\vartheta_{i}^{*}=19.6^{\circ} \mathrm{C}$.

Applying the thermovision in investigation from outside wall was shown a few degrees temperature difference (temperature on the cold surface joint $\vartheta_{e, s}^{*}=8.1^{\circ} \mathrm{C}$, temperature on the cold surface wall far from the joint $\vartheta_{e}^{*}=1.2^{\circ} \mathrm{C}$ the value of isothermal line near the joint $\vartheta_{e}^{*}=2.1^{\circ} \mathrm{C}$ ).

After the investigation described in this paper, the numerical calculations were performed, for boundary conditions, which were registered in the experiment.

Different pressure across the joint measurement in the experiment the following were for infiltration: $\Delta p=p_{e}-p_{i}=38 \mathrm{~Pa}$ and for exfiltration $\Delta p=p_{i}-p_{e}=30 \mathrm{~Pa}$. Convective heat transfer coefficient in the crack $\alpha_{s}$ accepted for $N u=7.6$ on the level $\alpha_{s}=95.5 \mathrm{~W} \cdot \mathrm{m}^{-2} \cdot \mathrm{K}^{-1}$.

The examined was wall characterized by thermal transmittance coefficient $k=$ $0.66 \mathrm{~W} \cdot \mathrm{m}^{-2} \cdot \mathrm{K}^{-1}$. Assuming that the standard airtightness coefficient cannot be bigger than $a_{z n}=0.135 \mathrm{~m}^{3} / \mathrm{h} . \mathrm{m}$. daPa ${ }^{0.67}$, calculation for the above data according to equation (2), where $I_{z}=3600^{-1} a_{2 n}(\Delta p / 10)^{0.67} \mathrm{~m}^{2} \cdot \mathrm{s}^{-1}$ give $\alpha_{p n}=0.058$ (infiltration) and $\alpha_{p n}=0.05 \mathrm{~W} \cdot \mathrm{m}^{-1} \cdot \mathrm{K}^{-1}$ (exfiltration). For natural volume flow rate through the crack, according to equation (2), $\alpha_{p}=0.189$ (infiltration) and $\alpha_{p}=0.151 \mathrm{~W} \cdot \mathrm{m}^{-1} \cdot \mathrm{K}^{-1}$ (exfiltration).

The calculations of the temperature of fields for infiltration conditions $\left(t_{i}=20\right.$ and $t_{\mathrm{e}}=-0.4^{\circ} \mathrm{C}$ ), firstly for the standard permeability $\left(\alpha_{p n}\right)$, secondly for the real permeability $\left(\alpha_{p}\right)$ may be compared with thermogram for the warm surface. The measurement of temperature differences between $\vartheta_{i}^{*}$ and $\vartheta_{i, s}^{*}$ equal to $9.9^{\circ} \mathrm{C}$ and exceeds 6.1 of the norm which is $3.8^{\circ} \mathrm{C}$ (for $\alpha_{p n}=0.058$ ). This means that our joint is not airtight. For real permeability $\left(\alpha_{p}=0.189\right)$, the difference temperature from the experiments is similar to difference received from numerical calculations $\left(\vartheta_{i}^{*}-\vartheta_{i, s}^{*} \rightarrow \vartheta_{i}-\vartheta_{i, s}=8.6^{\circ} \mathrm{C}\right)$. Compare curves in figures $1 \mathrm{~d}: 0$ - experiment; 1,2 - numerical calculation for standard $\alpha_{p n}$ and real $\alpha_{p}$, respectively.

Analogically, for exfiltration measurement of temperature differences read out from the thermogram, $\vartheta_{e, s}^{*}-\vartheta_{e}^{*}=6.9^{\circ} \mathrm{C}$, and the calculation of temperature of field for boundary condition (from the experiment: $t_{i}=21, t_{e}=0^{\circ} \mathrm{C}$ ) first for the standard permeability $\left(\alpha_{p n}=0.05\right)$, second for the real $\left(\alpha_{p}=0.151\right)$, give the difference of temperatures 2.8 and $7.4^{\circ} \mathrm{C}$, respectively. Compare the curves in figure $1 \mathrm{a}: 3$ - experiment; 4,5 - numerical calculation for standard $\alpha_{p m}$ and real $\alpha_{p}$, respectively.

In figure $1 \mathrm{~d}$ (curve 1), we see a sharp decreasing of the temperature on the warm surface of joint for standard $a_{z n}$. This means that standard airtightness joints of the external sandwich walls must be improved when there is the problem of surface condensation.

\section{Results}

The investigations and calculations show that the calculation model is physically adequate.

The routine investigation does not require command of air speed in the crack. The answer to the question of airtightness can be the result of the comparison temperature differences received from numerical calculation for exfiltration $\left(\vartheta_{e, s}-\vartheta_{e}\right)$ or for infiltration $\left(\vartheta_{i}-\vartheta_{i, s}\right)$, assuming standard 
tightness (for actual measurement difference pressure $\Delta p\left(\Delta p=p_{i}-p_{e}\right.$ or $\left.p_{e}-p_{i}\right)$ and difference temperatures, with the difference temperatures on the thermogram $\left(\vartheta_{e, s}^{*}-\vartheta_{e}^{*}\right)$ or $\left(\vartheta_{i}^{*}-\vartheta_{i, s}^{*}\right)$.

In investigations in situ, the difference pressure $\Delta p$ (across the joint) must be selected on the base simulation for actual temperature differences $\left(t_{i}-t_{\varepsilon}\right)$.

The results of the investigations confired the usefulness of the new thermal-pressure method for the airtightness joints testing.

\section{REFERENCES}

[1] PATTERSON (B.) and AXEN (B.). - Thermography, testing of the thermal insulation and airtightness of buildings. Swedish Council for Building Research, Stockholm, 1980.

[2] KOHONEN (R.) and VIRTANEN (M.). - Thermal coupling of leakage flows and heating load of buildings. ASHRAE Trans. 93, 197, p. 2303-2318.

[3] HOFFMAN (M.) and SCHWARTZ (B.). - Computation of steady and time-dependent temperature distribution for building elements. Building and Environment, 15, 1980, p. $63-72$.
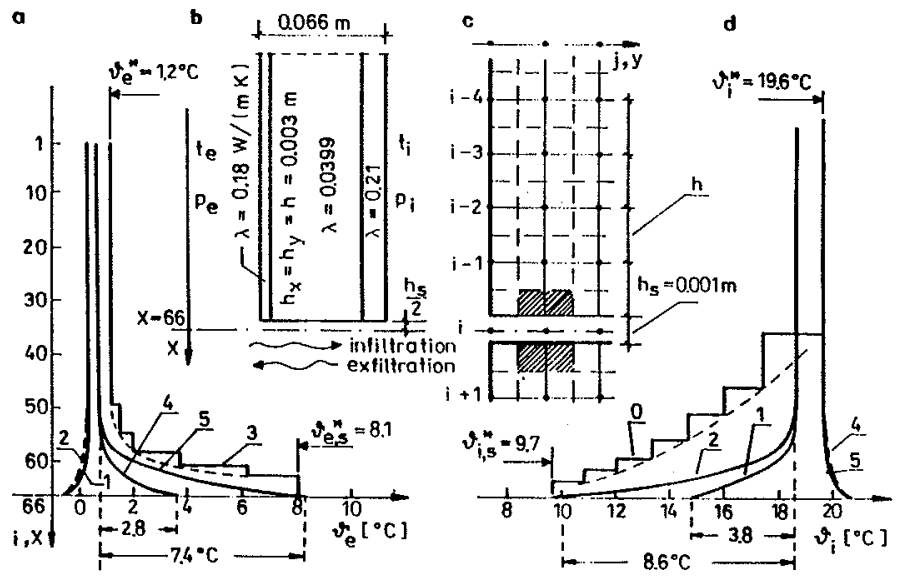

a - changes of the surface temperature on the cold side, b - model of the joint, $\mathbf{c}$ - network diagram, d - changes of the surface temperature on the warm side; for infiltration: 0 - experiment, 1, 2 - calculation for $\alpha_{p n}=0.058 W \cdot \mathrm{m}^{-1} \cdot \mathrm{K}^{-1}$ and $\alpha_{p}=0.189$, respectively; for exfiltration: 3 - experiment, 4,5 - calculation for $\alpha_{p n}=0.05$ and $\alpha_{p}=0.151$, respectively

Fig. 1. - Comparison of experimental and calculation values for infiltration $\left(t_{i}=20\right.$ and $t_{e}=-0.4^{\circ} \mathrm{C}$ ) and exfiltration $\left(t_{i}=21\right.$ and $\left.t_{e}=0^{\circ} \mathrm{C}\right)$ 